\section{In arm's way?}

\section{By Steve Edelson, Executive Editor}

Researchers at Genmab A/S have published a new hypothesis about how Tysabri natalizumab and certain other antibodies with IgG4 backbones elicit unwanted immune system effects. ${ }^{1}$ mAbs can be engineered to avoid the Fab transfer, and Genmab has one in discovery research.

IgG4 backbones are a logical choice for many therapeutic antibodies because this subclass of immunoglobulins is naturally antiinflammatory. For example, avoiding inflammation is clearly a goal for a multiple sclerosis drug like Tysabri natalizumab from Biogen Idec Inc. and Elan Corp. plc.

However, a known problem with IgG4 backbones is that they are flimsy, which can lead to half-life and stability issues. In vitro they have also been shown to undergo a process by which an IgG4 antibody swaps Fab arms with another IgG4 antibody. ${ }^{2}$ The Fab portion is responsible for binding antigens and is connected to the core segment of the antibody by a hinge region.

Now, a Genmab-led team has shown that IgG4-based therapeutic antibodies also exchange Fab arms with endogenous IgG4 antibodies in vivo (see Figure 1, "Toxicity and IgG4 antibody fragment exchange"). As a result, "the ability of a therapeutic IgG4 antibody to engage with plasma IgG4 could have substantial biological consequences," the team wrote in Nature Biotechnology.

The authors showed that in vitro and in mice, Tysabri underwent Fab-arm exchange with other IgG4 antibodies.

The findings also translated to humans. The authors analyzed blood samples from 16 Tysabri patients and found that 15 had Tysabri containing an endogenous type of Fab arm and thus different specificities for the drug's targets-integrin $\alpha_{4} \beta_{1}(C D 49 D / C D 29)$ and integrin $\alpha_{4} \beta_{7}$ receptors on certain types of white blood cells.

The group concluded that therapeutic antibodies can potentially undergo Fab-arm exchange "with pre-existing IgG4 antibodies with undesired specificity."

This process could provide an alternative mechanism for how Tysabri causes rare cases of progressive multifocal leukoencephalopathy (PML).

The first cases of PML arose in 2005 and prompted the withdrawal of Tysabri from the market shortly after it was launched. The drug was relaunched in 2006 with a boxed warning and a stringent monitoring program. Since then, there have been at least 11 additional cases of PML.

Tysabri prevents certain white blood cells from binding to vascular cell adhesion molecule-1 (VCAM-1), which is found on endothelial cells. The lymphocytes are therefore unable to leave the vasculature and are prevented from getting into the brain and attacking the myelin sheaths of neurons. It's been postulated that this mechanism could compromise immune surveillance for opportunistic infections, such as the John Cunningham virus (JCV) responsible for PML, the latent form of which is widespread in the general population. ${ }^{3}$

Based on the findings in the Nature Biotechnology article, the authors proposed that "circulating IgG4 antibodies against JCV might recombine with natalizumab by Fab-arm exchange. The JCV/VLA-4 bispecific antibodies generated could then mediate the capture of JCV by infiltrating activated leukocytes and thus facilitate the transport of JCV into the central nervous system."

A simple pharmacokinetic model of Fab-arm exchange did show that undesired cross-linking is "unlikely under normal conditions," according to the researchers. However, they added, "we cannot exclude the possibility that cross-linking at a low level or under certain pathological conditions may still be sufficient to induce undesired biological effects."

\section{No exchanges}

Given the potential for Fab-arm exchange to adversely affect a therapeutic antibody's safety or efficacy profile, it's clear that companies should be thinking about how to prevent the process. The good news is that one marketed antibody with an IgG4 backbone-Mylotarg gemtuzumab from UCB Group and Wyeth-provides a road map for eliminating Fab-arm exchange.

Mylotarg, a humanized antibody against CD33 that delivers the toxin calicheamicin, is approved to treat $\mathrm{CD} 33^{+}$acute myelogenous

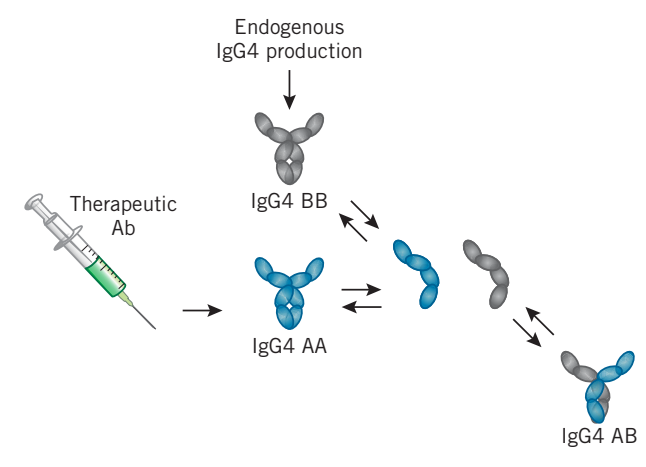

Figure 1. Toxicity and IgG4 antibody fragment exchange. The exchange of Fab arms between therapeutic and endogenous IgG4 antibodies can result in a mAb with specificity for both its intended target and whatever target the endogenous antibody binds to. For example, a therapeutic IgG4 antibody like Tysabri natalizumab (IgG4 AA in figure), which targets integrin $\alpha_{4}$ (ITGA4; VLA-4; CD49D), could exchange its Fab arms with endogenously produced IgG4 in humans (IgG4 BB in figure). The authors suggest that the resulting bispecific antibodies (IgG4 AB in figure) could potentially cause unwanted side effects. 
leukemia (AML) in patients with first relapse who are 60 or older and who are not considered candidates for other cytotoxic chemotherapy. Because the antibody kills cells by delivering a toxin, the IgG4 backbone was a logical choice to avoid recruiting-and thus destroying-immune cells.

Because IgG4 backbones are notoriously delicate, Mylotarg was engineered to have a mutation (S228P) in its core-hinge region. "The mutation was done from a manufacturing point of view-to make the product more homogeneous," said Paul Parren, SVP of research and preclinical development at Genmab and corresponding author on the Nature Biotechnology article. "So it was sort of serendipity, because the mutation also prevents Fab-arm exchange."

Indeed, the authors of the paper reported that Mylotarg's corehinge mutation "blocks Fab-arm exchange to undetectable levels both in vitro and in a mouse model."

Genmab has taken a different approach to increasing the stability of IgG4 backbones and preventing Fab-arm exchange: getting rid of the hinge region entirely. The company's UniBody technology essentially splits Y-shaped antibodies down the middle, leaving a half-sized antibody fragment. ${ }^{4}$
The resulting molecules, said Parren, "are monovalent, have long half-lives and do not have Fab exchange activity."

Genmab's most advanced UniBody is in discovery research for undisclosed indications.

Biogen Idec and Elan declined to comment.

Edelson, S. SciBX 2(32); doi:10.1038/scibx.2009.1231

Published online Aug. 20, 2009

\section{REFERENCES}

1. Labrijn, A. et al. Nat. Biotechnol.; published online July 20, 2009; doi:10.1038/nbt.1553

Contact: Paul Parren, Genmab A/S, Copenhagen, Denmark e-mail: p.parren@genmab.com

2. van der Neut Kolfschoten, M. et al. Science 317, 1554-1557 (2007)

3. Maggos, C. BioCentury 13(11), A1-A5; March 7, 2005

4. Ward, M. BioCentury 14(47), A16; Oct. 23, 2006

COMPANIES AND INSTITUTIONS MENTIONED

Biogen Idec Inc. (NASDAQ:BIIB), Cambridge, Mass.

Elan Corp. plc (NYSE:ELN), Dublin, Ireland

Genmab A/S (CSE:GEN), Copenhagen, Denmark

UCB Group (Euronext:UCB), Brussels, Belgium

Wyeth (NYSE:WYE), Madison, N.J. 